\title{
Conjugations of Arithmetic Automorphic Function Fields
}

\author{
Kuang-yen Shih * \\ Department of Mathematics, University of Michigan, Ann Arbor, Mi 48109, USA
}

In [1], Doi and Naganuma showed that the conjugation of a Shimura curve is again a Shimura curve. The present paper deals with the generalization of their result.

Consider in general a reductive algebraic group $G$ defined over $\mathbf{Q}$. Let $G^{u}$ be the semi-simple part of $G$. Assume that $G_{\mathbf{R}}^{u}$ modulo a maximal compact subgroup defines a bounded symmetric domain $\mathscr{H}$, and that a system of canonical models (in the sense of Shimura $[11,2.13])$ for the quotients of $\mathscr{H}$ by the arithmetic subgroups $\Gamma_{X}$ of $G$ exists. Let $\left\{V_{X}, \phi_{X}, J_{X W}(u)\right\}$ be such a system. Take an arbitrary automorphism $\tau$ of the complex number field $\mathbf{C}$, and conjugate all the $V_{X}$ 's and $J_{X W}(u)$ 's by $\tau$. Then one expects that $\left\{V_{X}^{\tau}, \tilde{\phi}_{X}, J_{X W}(u)^{\tau}\right\}$ with suitable $\tilde{\phi}_{X}$ 's forms a system of canonical models for some reductive group $G_{1}$.

In this paper we show that this is the case if $G$ is the type of groups investigated by Shimura in [11]. The corresponding group $G_{1}$ is defined explicitly in 1.2 , and the precise statement of the result is given as Theorem 1.3.

In Shimura's construction, for special $\Gamma_{X}$ the model $V_{X}$ is first realized as a subvariety of a moduli-variety $V_{\Omega}$ for some PEL-type $\Omega$. Consider $V_{X}^{\tau}$ as embedded in $V_{\Omega^{\prime}}^{\tau}$. It is known that the conjugate variety $V_{\Omega}^{\tau}$ is isomorphic to the moduli-variety $V_{\Omega^{\prime}}$ of another PEL-type $\Omega^{\prime}$. The relations between $\Omega$ and $\Omega^{\prime}$ are provided by Shimura's work [6]. One of our main task then is to prove that the isomorphism of $V_{\Omega}^{z}$ to $V_{\Omega^{\prime}}$ induces an isomorphism of $V_{X}^{\tau}$ to $V_{X_{1}}$ for some arithmetic subgroup $\Gamma_{X_{1}}$ of $G_{1}$. This can be achieved by studying the isolated fixed points of $G_{\mathbf{Q}}$ and $G_{1 \mathbf{Q}}$. We carry out these considerations in Sections 2 and 3.

The same functorial property also holds for the models constructed by Miyake in [3]. In Section 4 we deal with this case briefly. We shall not give the proof, because the argument is similar to, and actually simpler than, the one presented in this paper.

We assume the reader is familiar with Shimura's work [10] and [11], which will be quoted respectively as $[\mathrm{A}]$ and $[\mathrm{C}]$ hereafter.

* Partially supported by NSF grant MPS 75-07948 


\section{Notations}

We adopt the notations of [C], of which some are recalled here.

The multiplicative group of an associative $\operatorname{ring} S$ with identity is denoted by $S^{\times}$. For an algebraic group $G$ defined over $\mathbf{Q}, G_{\mathbf{A}}$ denotes its adelization. The finite part and infinite part of $G_{A}$ are denoted by $G_{0}$ and $G_{\infty}$ respectively. Denote the identity component of $G_{\infty}$ by $G_{\infty+}$, and put $G_{\mathrm{A}_{+}}=G_{0} G_{\infty+}$.

For an algebraic number field $F, \mathfrak{x}_{F}$ denotes the ring of integers of $F, F_{\mathrm{ab}}$ the abelian closure of $F$, and $F_{c}$ the closure of $F^{\times} F_{\infty+}^{\times}$in the idele group $F_{\mathrm{A}}^{\times}$.

\section{The Main Theorem}

1.1. Let $F$ be a totally real algebraic number field of degree $g$, and $B$ a quaternion algebra over $F$. Let $\tau_{1}, \ldots, \tau_{g}$ be the $g$ distinct isomorphisms of $F$ into $R$ arranged in such a way that $B$ is unramified at $\tau_{1}, \ldots, \tau_{r}$, and ramified at all other infinite places. Denote the discriminant of $B$ over $F$ by $D(B / F)$.

Assume $r>0$. Define an algebraic group $G$ over $\mathbf{Q}$ so that the $\mathbf{Q}$-rational points of $G$ are

$$
G_{\mathbf{Q}}=\left\{\alpha \in G L_{n}(B) \mid \alpha \cdot{ }^{t} \alpha^{i}=v(\alpha) 1_{n} \text { with } v(\alpha) \in F^{\times}\right\},
$$

where $\imath$ denotes the main involution of $B$ and ${ }^{t} \alpha$ the transpose of $\alpha$. The semi-simple part of $G$ is

$$
G^{u}=\{\alpha \in G \mid v(\alpha)=1\},
$$

and $G_{\mathbf{R}}^{u}$ modulo a maximal compact subgroup defines a bounded symmetric domain $\mathscr{H}$ which can be identified with $\mathscr{H}_{n}^{r}, r$ copies of Siegel's upper half space $\mathscr{H}_{n}$ of degree $n$.

Consider a representation $\theta$ of $F$ equivalent to $\sum_{v=1}^{r} \tau_{v}$. Let $\left(F^{\prime}, \theta^{\prime}\right)$ be the reflex of $(F, \theta)$ and $\lambda=\operatorname{det} \theta^{\prime}$ (see $[\mathrm{CI}, \S 1]$ for notation). Then $\lambda$ is a homomorphism of $F^{\prime \times}$ to $F^{\times}$. Put

$$
\overline{\mathscr{G}}_{+}=\left\{x \in G_{\mathbf{A}+} \mid v(x) \in \lambda\left(F_{\mathbf{A}}^{\prime \times}\right) F_{c}\right\} .
$$

Define a group extension $\mathfrak{A}$ of $\mathfrak{A}^{1}=\overline{\mathscr{G}}_{+} / F_{c} G_{\infty+}$ and a subgroup $A_{+}$of $\mathfrak{A}$ as in [CII, §4]. Let $\xi^{*}$ be the subfield of $F_{\mathrm{ab}}^{\prime}$ defined in [CII, 3.10], and $\rho$ the homomorphism from $\mathcal{A}$ to $\operatorname{Gal}\left(\mathfrak{f}^{*} / F^{\prime}\right)$ defined in [CII, 4.7]. The kernel of $\rho$ is the closure of $A_{+}$. Consider the set 3 of all open compact subgroup of $\mathfrak{A}$. For every $X \in \mathcal{Z}, \Gamma_{X}=A_{+} \cap X$ acts on $\mathscr{H}$ properly discontinuously and $\mathscr{H} / \Gamma_{X}$ has finite measure. The subgroup $\rho(X)$ of $\operatorname{Gal}\left(\mathfrak{f}^{*} / F^{\prime}\right)$ corresponds to a subfield of $\mathfrak{f}^{*}$, which we denote by $k_{x}$.

As shown in [C], for every $X \in \mathcal{Z}$ there is a model $\left(V_{X}, \phi_{X}\right)$ of $\mathscr{H} / \Gamma_{X}$ with $V_{X}$ rational over $k_{X}$, and for $u \in \mathfrak{A}, X, W \in 3$ such that $u X u^{-1} \subset W$, there is a morphism $J_{W X}(u)$ of $V_{X}$ onto $V_{W}^{\rho(u)}$ rational over $k_{X}$. Furthermore the system $\left\{V_{X}, \phi_{X}, J_{W X}(u),(X, W \in 3 ; u \in \mathfrak{A})\right\}$ enjoys the properties stated in [CII, Theorem 5.2]. We call it a canonical system of models associated with $G$. 
1.2. Let $\tau$ be an automorphism of $\mathbf{C}$. By a well-known Theorem of Hasse, there is a quaternion algebra $B_{1}$ over $F$, unique up to $F$-linear isomorphism, such that $B_{1}$ is unramified at $\tau_{1} \tau, \ldots, \tau_{r} \tau$, ramified at all other infinite places, and $D\left(B_{1} / F\right)$ $=D(B / F)$. Consider the algebraic group $G_{1}$ over $\mathbf{Q}$ whose $\mathbf{Q}$-rational points are

$$
G_{1 \mathbf{Q}}=\left\{\alpha \in G L_{n}\left(B_{1}\right) \mid \alpha \cdot{ }^{t} \alpha^{2}=\nu_{1}(\alpha) 1_{n} \text { with } v_{1}(\alpha) \in F^{\times}\right\} .
$$

Define the counterparts $\lambda_{1}, \mathfrak{H}_{1}, \mathfrak{F}_{1}^{*}, \rho_{1}, \mathfrak{Z}_{1}$, etc. of $\lambda, \mathfrak{H}, \mathfrak{t}^{*}, \rho, 3$, etc. Note that the representation $\theta_{1}$ of $F$ associated with $B_{1}$ is equivalent to $\sum_{v=1}^{r} \tau_{v} \tau \sim \theta^{\tau}$. Denote the reflex of $\left(F, \theta_{1}\right)$ by $\left(F_{1}^{\prime}, \theta_{1}\right)$. Then it follows from Lemma 1.5 proved below that $F_{1}^{\prime}$ $=F^{\prime \tau}, \lambda_{1}\left(x^{\tau}\right)=\lambda(x)$ for $x \in F^{\prime}$, and $\mathfrak{F}_{1}^{*}=\mathfrak{f}^{* \tau}$. Let $\left\{V_{X_{1}}, \phi_{X_{1}}, J_{W_{1} X_{1}}\left(u_{1}\right),\left(X_{1}, W_{1} \in \mathcal{Z}_{1}\right.\right.$; $\left.\left.\mathrm{u}_{1} \in \mathfrak{Q}_{1}\right)\right\}$ be a system of canonical models associated with $G_{1}$.

1.3. Theorem. There is a topological isomorphism $\mathfrak{u}$ of $\mathfrak{A}$ to $\mathfrak{A}_{1}$, which is locally algebraic, with the following properties. For $X_{1} \in \mathfrak{Z}_{1}$ and $u_{1} \in \mathfrak{H}_{1}$, put $X=\mathfrak{u}^{-1}\left(X_{1}\right)$ and $u=u^{-1}\left(u_{1}\right)$. Then $k_{X_{1}}=k_{X}^{\tau}, \tau \rho_{1}\left(u_{1}\right)=\rho(u) \tau$ on $\mathfrak{f}^{*}$, and for each $X_{1} \in 3_{1}$, there is a biregular isomorphism $\psi_{X}$ of $V_{X_{1}}$ to $V_{X}^{\tau}$ rational over $k_{X}^{\tau}$ such that

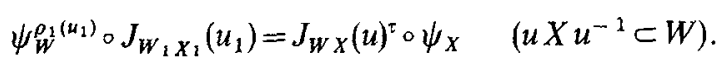

Remark. We have $\tau=$ id. on $F^{\prime}$ if and only if $\left\{\tau_{1}, \ldots, \tau_{r}\right\}=\left\{\tau_{1} \tau, \ldots, \tau_{r} \tau\right\}$. In this case we have $B_{1}=B$ and $G_{1}=G$. Since $\rho: \mathfrak{Q} \rightarrow \operatorname{Gal}\left(\mathfrak{f}^{*} / F^{\prime}\right)$ is surjective, there exists $u \in \mathfrak{Q}$ such that $\rho(u)=\tau$ on $\mathfrak{f}^{*}$. Define $\mathfrak{H}(x)=u^{-1} x u$ for $x \in \mathfrak{U}$. Then $\mathfrak{u}$ satisfies the conditions of Theorem 1.3. Therefore the theorem is trivial in the case where $\tau=\mathrm{id}$. on $F^{\prime}$.

1.4. For $X \in \mathcal{3}$, let $K_{X}$ be the field of all functions on $V_{X}$ rational over $k_{X}$, and put

$$
\begin{aligned}
\mathscr{K}_{X} & =\left\{f \circ \phi_{X} \mid f \in K_{X}\right\}, \\
\mathscr{K} & =\bigcup_{X \in \mathcal{B}} \mathscr{K}_{X} .
\end{aligned}
$$

We call $\mathscr{K}$ the arithmetic automorphic function field associated with $G$. For $u \in \mathfrak{A}$ and $f \in K_{X}$, put

$$
\left(f \circ \phi_{X}\right)^{\omega(u)}=f^{\rho(u)} \circ J_{X W}(u) \circ \phi_{W},
$$

where $W=u^{-1} X u$. Then $\omega$ is a homomorphism of $\mathfrak{A}$ to Aut $\left(\mathscr{K} / F^{\prime}\right)$. Let $\mathscr{K}_{1}$ be the field of arithmetic automorphic functions associated with $G_{1}$, and $\omega_{1}: \mathfrak{U}_{1} \rightarrow \operatorname{Aut}\left(\mathscr{K}_{1} / F_{1}^{\prime}\right)$ the homomorphism corresponding to $\omega$. In terms of arithmetic automorphic function fields, we can restate Theorem 1.3 as follows.

Theorem. Let $\mathfrak{u}: \mathfrak{A} \rightarrow \mathfrak{A}_{1}$ be as in Theorem 1.3. Then there is an isomorphism $\pi: \mathscr{K} \rightarrow \mathscr{K}_{1}$ extending $\tau: \mathfrak{f}^{*} \rightarrow \mathfrak{I}_{1}^{*}$ such that $\pi \omega_{1}\left(u_{1}\right)=\omega(u) \pi$ for $u \in \mathfrak{A}$ and $u_{1}$ $=\mathfrak{u}(u) \in \mathfrak{Q}_{1}$.

1.5. Lemma. Let $E$ be an algebraic number field and $\Psi$ a $\mathbf{Q}$-linear representation of $E$ by complex matrices. For an automorphism $\tau$ of $\mathrm{C}$, let $\Psi_{1}$ be a representation of $E$ equivalent to $\Psi^{\prime}$. Denote the reflexes of $\left(E, \Psi^{\prime}\right)$ and $\left(E, \Psi_{1}\right)$ by $\left(E^{\prime}, \Psi^{\prime}\right)$ and $\left(E_{1}^{\prime}, \Psi_{1}^{\prime}\right)$ respectively. Then $E_{1}^{\prime}=\left(E^{\prime}\right)^{x}$ and $\Psi_{1}^{\prime}$ is equivalent to the representation $\Phi$ of $E_{1}^{\prime}$ given by $\Phi\left(x^{\tau}\right)=\Psi^{\prime}(x)\left(x \in E^{\prime}\right)$. 
Proof. By definition, $E_{1}^{\prime}$ is generated over $\mathbf{Q}$ by $\left\{\operatorname{tr} \Psi_{1}(x) \mid x \in E\right\}$. Since $\operatorname{tr} \Psi_{1}(x)$ $=(\operatorname{tr} \Psi(x))^{\tau}$, we have $E_{1}^{\prime}=\left(E^{\prime}\right)^{\tau}$. To show $\Psi_{1}^{\prime}$ is equivalent to $\Phi$, take an $\left(E, E^{\prime}\right)$-module of type $(E, \Psi)$ and of type $\left(E^{\prime}, \Psi^{\prime}\right)[C I, 1.1]$. Introduce an $\left(E, E_{1}^{\prime}\right)$-module structure on $V$ as follows: For $a \in E, b \in E_{1}^{\prime}$ and $x \in V$, put $(a \otimes b) x=a x c$, where $c$ is the element of $E^{\prime}$ such that $c^{\tau}=b$. Call this $\left(E, E_{1}^{\prime}\right)$-module $V_{1}$. Then $V_{1}$ is of type $\left(E, \Psi_{1}\right)$ as well as of type $\left(E_{1}^{\prime}, \Phi\right)$. Therefore $\Psi_{1}^{\prime} \sim \Phi$.

Remark. The above Lemma can be regarded as dual to Proposition 12 of [4].

1.6. Let $B^{*}$ be the quaternion algebra over $F^{\tau}$ such that $D\left(B^{*} / F^{\tau}\right)=D(B / F)^{\tau}$ and such that $B^{*}$ is unramified at $\tau^{-1} \tau_{v} \tau$ for $v=1, \ldots, r$, and ramified for $v=r+1, \ldots, g$. It is easy to see that the algebraic group defined in terms of $G L_{n}\left(B^{*}\right)$ is isomorphic to $G_{1}$ over $\mathbf{Q}$. Especially, if $F^{\tau}=F, D(B / F)^{\tau}=D(B / F)$ and $\left\{\tau^{-1} \tau_{1} \tau, \ldots, \tau^{-1} \tau_{r} \tau\right\}$ $=\left\{\tau_{1}, \ldots, \tau_{r}\right\}$, then $G_{1}$ is isomorphic to $G$. Under this situation we have $F^{\prime \tau}=F^{\prime}$. The isomorphism $\mathfrak{u}$ of Theorem 1.3 can be considered as an automorphism of $\mathfrak{A}$. If $\tau \neq \mathrm{id}$. on $F^{\prime}$, then $\mathfrak{u}$ is not an inner automorphism. It would be interesting, as suggested by the Corollary in the introduction of [1], to see under what conditions we can reduce the fields of definition of $V_{X}$ 's. The author hopes to discuss this and other implications of the result on some other occasion.

\section{Construction of $\mathfrak{u}$}

2.1. Let $K$ be a totally imaginary quadratic extension of $F$. For each $v=1, \ldots, g$, fix an extension of $\tau_{v}$ to $K$ and denote it again by $\tau_{v}$. By Hasse's Theorem on central simple algebras, there is a quaternion algebra $L$ over $K$ which is isomorphic to both $B \otimes_{F} K$ and $B_{1} \otimes_{F} K$ over $K$. Denote the main involution of $L$ by $l$. Then there are a positive involution $\rho$ on $L$ and two invertible elements $v$ and $v_{1}$ of $L$ such that $v^{\rho}=$ $-v, v_{1}^{\rho}=-v_{1}$, and

$$
\begin{gathered}
B \cong\left\{x \in L \mid x^{l}=v x^{\rho} v^{-1}\right\}, \\
B_{1} \cong\left\{x \in L \mid x^{\imath}=v_{1} x^{\rho} v_{1}^{-1}\right\},
\end{gathered}
$$

see $[8,7.2]$. Via these isomorphisms we regard $B$ and $B_{1}$ as $F$-subalgebras of $L$. Put $T=v 1_{n}, S=v^{-1} 1_{n}$ and $\kappa=-\left(v v^{i}\right)^{-1}$. Then $T, S \in G L_{n}(L), \kappa \in F^{\times}$and

$$
{ }^{t} T^{\rho}=-T, \quad S^{i \rho} S=\kappa 1_{n}, \quad T \cdot{ }^{i} S^{\rho}=-S^{i \rho} T^{i \rho}
$$

[A, Prop. 6.2]. We assume Conditions (6.3.7) and (6.3.8) of [A] for $\kappa$ and $T$. Similarly, put $T_{1}=v_{1} 1_{n}, S_{1}=v_{1}^{-1} 1_{n}$ and $\kappa_{1}=-\left(v_{1} v_{1}^{l}\right)^{-1}$. We also assume for $T_{1}$ and $\kappa_{1}$ the conditions corresponding to $[\mathrm{A},(6.3 .7),(6.3 .8)]$.

Let $\not h$ be a finite prime of $F$. Denote the completion of $F$ at $\not h$ by $F_{\not}$. For an algebra $A$ over $F$, put $A_{p h}=A \otimes_{F} F_{p}$.

2.2. Proposition. For every finite prime $\not h$ of $F$, there is $a \in K_{\not h}^{\times}$such that $\kappa / \kappa_{1}=a a^{p}$.

Proof. Since $B_{\not k}$ and $B_{1 \not h}$ are $F_{n}$-isomorphic, there is $b$ in $L_{\not h}^{\times}$such that $B_{1 \not k}=b B_{\not h} b^{-1}$. It follows that

$$
B_{1 \not}=\left\{x \in L_{\not} \mid x^{2}=\left(b v b^{\rho}\right) x^{\rho}\left(b v b^{\rho}\right)^{-1}\right\} .
$$


We also have $\left(b v b^{\rho}\right)^{\rho}=-b v b^{\rho}$. Hence $v_{1}=c b v b^{\rho}$ for some $c \in F_{k}^{\times}$. Denote the reduced norm from $L$ to $K$ by $N$. Then it is easy to see that $\kappa=a a^{\rho} \kappa_{1}$ with $a=c N(b)$.

2.3. Proposition. For every finite prime $\not h$ of $F$, there are $c \in F_{p}^{\times}$and $\alpha \in G L_{n}\left(L_{k}\right)$ such that $T_{1}=c \alpha T \cdot{ }^{t} \alpha^{\rho}$.

Proof. Let the notation be as in the proof of Proposition 2.2. Put $\alpha=b 1_{n}$. Then we have $T_{1}=c \alpha T \cdot{ }^{i} \alpha^{p}$.

2.4. Let

$$
\mathscr{P}=\left\{z \in M_{n}(\mathbf{C}) \mid 1-{ }^{t} \bar{z} z \text { is positive definite }\right\}
$$

and

$$
\mathscr{D}_{n}=\left\{\left.z \in \mathscr{S}\right|^{t} z=z\right\} .
$$

Define an algebraic group $G^{*}$ over $\mathbf{Q}$ so that the $\mathbf{Q}$-rational points of $G^{*}$ are the group of similitudes of $T$ :

$$
G_{\mathbf{Q}}^{*}=G(T)_{\mathbf{Q}}=\left\{\alpha \in G L_{n}(L) \mid a T \cdot{ }^{\prime} \alpha^{p}=v(\alpha) T \text { with } v(\alpha) \in F^{\times}\right\} .
$$

Then $G_{\mathbf{R}+}^{*}$ acts on $\mathscr{S}^{r}$ as described in $[\mathrm{A}, 6.5]$. As shown in $[\mathrm{A}, 6.6]$ there are maps

$i: G \rightarrow G^{*}, \quad$ a Q-rational injection,

$i^{\prime}: B^{n} \rightarrow L^{n}, \quad$ a $B$-linear injection,

$j: \mathscr{H}_{n}^{r} \rightarrow \mathscr{D}_{n}^{r} \subset \mathscr{P}^{r}, \quad$ a holomorphic bijection,

such that $v(i(\alpha))=v(\alpha), j(\alpha(z))=i(\alpha)(j(z)), i^{\prime}(x \alpha)=i(x) i^{\prime}(\alpha)$ for $\alpha \in G_{\mathbf{Q}+}, \quad z \in \mathscr{H}_{n}^{r}$ and $x \in B^{n}$. The image of $G$ under $i$ is

$$
i(G)=\left\{\alpha \in G^{*} \mid \alpha^{i \rho} S=S \alpha\right\} .
$$

Using $T_{1}$ and $S_{1}$ instead of $T$ and $S$, we define the counterparts of $G^{*}, i, i^{\prime}$ and $j$, and denote them by $G_{1}^{*}, i_{1}, i_{1}^{\prime}$ and $j_{1}$ respectively.

2.5. From $B$ we can construct a representation $\Phi$ of $L_{\mathbf{R}}$ by complex matrices as in [A, 6.4]. Similarly, define a representation $\Phi_{1}$ of $L_{\mathbf{R}}$, using $B_{1}$ instead. It is easy to see that $\Phi_{1}$ is equivalent to $\Phi^{\tau}$. Let $\left(H, \Phi^{\prime}\right)$ be the reflex of $(K, \Phi)$. Choose an ample $\mathfrak{r}_{F}$-lattice $\mathfrak{m}$ in $B^{n}$ in the sense of $[\mathrm{A}, 3.7]$, and put $\mathfrak{M}=\mathrm{r}_{K} \cdot \mathfrak{m}$. Then $\mathfrak{M}$ is an $\mathbf{r}_{K}$-lattice in $L^{n}$. Replacing $v$ by $c v$ for suitable $c \in F_{+}^{\times}$if necessary, we can assume $\operatorname{tr}_{L / \mathbf{Q}}(T(\mathfrak{M}, \mathfrak{M})$ ) $=\mathrm{Z}$. Form the PEL-type $\Omega=(L, \Phi, \rho ; T, \mathfrak{M})$. Then $\Omega^{x}=\left(L, \Phi_{1}, \rho ; T^{*}, \mathfrak{M}^{*}\right)$, where $T^{*} \in M_{n}(L)$ and $\mathfrak{M}^{*}$ is a lattice in $L^{n}$. By [6I, Proposition 1.11], for every finite prime $\not p$ of $F$, there are $c \in F_{p h}^{\times}$and $\alpha \in G L_{n}\left(L_{p}\right)$ such that $T^{*}=c \alpha T \cdot{ }^{t} \alpha^{\rho}$. (As already noted in [1], the original condition that $\tau=$ id. on $H$ is not necessary.)

Note that both $T^{*}$ and $T_{1}$ have signature $(n, n)$ at an infinite prime corresponding to $\tau_{v} \tau(v=1, \ldots, r)$, and $(2 n, 0)$ at other infinite primes of $F$. Next, combining Proposition 2.3 with the observation made at the end of the last paragraph, we see that for every finite prime $\not p$ of $F$, there are $c_{k} \in F_{k}^{\times}$and $\alpha_{k} \in G L_{n}\left(L_{k}\right)$ such that $\alpha_{k} T^{*} \cdot{ }^{t} \alpha_{k}^{\rho}=c_{k} T_{1}$. Hence by a Theorem of Landherr [2], there are $c \in F_{+}^{\times}$and $\alpha \in G L_{n}(L)$ such that $\alpha T^{*} \cdot{ }^{t} \alpha^{\rho}=c T_{1}$. 
Replace $v_{1}$ by $c v_{1}$, hence $T_{1}$ by $c T_{1}$. Then $\Omega^{\mathrm{t}}=\left(L, \Phi_{1}, \rho ; T^{*}, \mathfrak{M}^{*}\right)$ is equivalent to $\left(L, \Phi_{1}, \rho ; T_{1}, \mathfrak{M}^{*} \alpha^{-1}\right)$. Put $\mathfrak{M}_{1}=\mathfrak{M}^{*} \alpha^{-1}$. Then we can assume

$$
\Omega=(L, \Phi, \rho: T, \mathfrak{M}) \quad \text { and } \quad \Omega^{\tau}=\left(L, \Phi_{1}, \rho ; T_{1}, \mathfrak{M}_{1}\right) .
$$

2.6. Take a totally imaginary quadratic extension $P$ of $F$ different from $K$ such that $B \otimes_{F} P$ is isomorphic to $M_{2}(P)$. Put $Y=M_{n}(P)$. Denote the complex conjugation on $P$ by $\rho$. For $a \in Y$, put $a^{\delta}={ }^{t} a^{\rho}$. Then $\delta$ is a positive involution on $Y$. By [A, 4.7] there is an $F$-linear isomorphism $f$ of $Y$ into $M_{n}(B)$ such that $f\left(a^{\delta}\right)={ }^{t} f(a)^{x}$ for all $a \in Y$. Let $z \in \mathscr{H}$ be the fixed point of $(Y, P, \delta, f)[\mathrm{A},(4.7 .5)]$. We use $(Y, P, \delta, f)$ to define a representation $\Psi$ of $P$ as in $[\mathrm{A}, 4.9]$. The representation $\Psi$ is equivalent to $\sum_{v=1}^{r} \chi_{v}$, where $\chi_{v}(v=1, \ldots, r)$ is one of the extensions of $\tau_{v}$ to $P$. For $v=r+1, \ldots, g$, let $\chi_{v}$ be any extension of $\tau_{v}$ to $P$.

By Hasse's Theorem, $B_{1} \otimes_{F} P$ is also isomorphic to $M_{2}(P)$. Therefore there is an $F$-linear isomorphism $f_{1}$ of $Y$ into $M_{n}\left(B_{1}\right)$ such that $f_{1}\left(a^{\delta}\right)={ }^{t} f_{1}(a)^{t}$. Let $z_{1} \in \mathscr{H}$ be the fixed point of $\left(Y, P, \delta, f_{1}\right)$. Let $\Psi_{1}$ be the representation of $P$ constructed from $\left(Y, P, \delta, f_{1}\right)$ as $\Psi$ was from $(Y, P, \delta, f)$. Conjugating $f_{1}$ by a suitable element of $G L_{n}\left(B_{1}\right)$, we may assume that $\Psi_{1}$ is equivalent to $\Psi^{\tau}$.

2.7. Let $\Sigma(\Omega)=\left\{\mathscr{Q}_{w} \mid w \in \mathscr{P}^{r}\right\}$ be an analytic family of PEL-structures of type $\Omega$ with parametrizing function $\mathfrak{y}(x, w)$, see $[\mathrm{A}, 6.4]$. Let $(Y, P, \delta, f)$ be as in 2.6. Put $Z$ $=L \otimes_{F} Y \cong M_{2 n}(K P)$, and regard $L^{n}$ as a $Z$-module by setting $x(a \otimes b)=a x(i(f(b)))$ for $a \in L, b \in Y$ and $x \in L^{n}$. Let $z \in \mathscr{H}$ be the fixed point of $(Y, P, \delta, f)$ and $y=j(z) \in \mathscr{S}^{r}$. Consider the structure $\mathscr{Q}_{y}=\left(A_{y}, \mathscr{C}_{y}, \theta_{y}\right)$. We can extend $\theta_{y}$ to an anti-isomorphism $\theta^{*}$ of $Z$ into $\operatorname{End}_{\mathbf{Q}}\left(A_{y}\right)$ as in [A, 6.7-6.9]. Define a representation $\Xi$ of $Z$ so that

$$
\begin{aligned}
& \Xi \sim 2 \Psi+\sum_{v=r+1}^{g}\left(\chi_{v}+\chi_{v} \rho\right) \quad \text { on } P, \\
& \Xi \sim \sum_{v=1}^{r}\left(\tau_{v}+\tau_{v} \rho\right)+2 \sum_{v=r+1}^{g} \tau_{v} \text { on } K,
\end{aligned}
$$

see $[\mathrm{A}, 6.8,6.9]$. For $a, a^{\prime} \in L^{n}$ put $U\left(a, a^{\prime}\right)=\operatorname{tr}_{L / \mathbf{Q}}\left(T\left(a, a^{\prime}\right)\right)$. Then $\mathscr{Q}^{*}=\left(A_{y}, \mathscr{C}_{y}, \theta^{*}\right)$ is of type $(Z, \Xi, \mathfrak{M}, U)$ in the sense of $[\mathrm{CI}, 4.1]$.

Let $\left(Y, P, \delta, f_{1}\right)$ and $z_{1}$ be as in 2.6, and put $y_{1}=j_{1}\left(z_{1}\right)$. Denote $\Omega^{\tau}$ $=\left(L, \Phi_{1}, \rho ; T_{1}, \mathfrak{M}_{1}\right)$ by $\hat{\Omega}$. Let $\Sigma(\hat{\Omega})=\left\{\hat{\mathscr{Q}}_{w} \mid w \in \mathscr{S}^{r}\right\}$ be an analytic family of PELstructures of type $\hat{\Omega}$ with parametrizing function $\mathfrak{y}_{1}(x, w)$. We can extend $\hat{\mathscr{Q}}_{y_{1}}$ $=\left(\hat{A}_{y_{1}}, \hat{\mathscr{C}}_{y_{1}}, \hat{\theta}_{y_{1}}\right)$ to a structure $\hat{\mathscr{Q}}^{*}=\left(\hat{A}_{y_{1}}, \hat{\mathscr{C}}_{y_{1}}, \hat{\theta}^{*}\right)$ of type $\left(Z, \Xi_{1}, \mathfrak{M}_{1}, U_{1}\right)$. Here we regard $L^{n}$ as a $Z$-module by setting $x(a \otimes b)=a x\left(i_{1}\left(f_{1}(b)\right)\right)$. $\Xi_{1}$ is a representation of $Z$ defined in the same way as $\Xi$ was defined in the last paragraph. And $U_{1}\left(a, a^{\prime}\right)$ $=\operatorname{tr}_{L / \mathbf{Q}}\left(T_{1}\left(a, a^{\prime}\right)\right)$ for $a, a^{\prime} \in L^{n}$. Comparing the restriction of $\Xi_{1}$ and $\Xi^{\boldsymbol{r}}$ to both $K$ and $P$, we see that $\Xi_{1}$ is equivalent to $\Xi^{\tau}$.

We show that if the parametrizing function $\mathfrak{y}_{1}$ is chosen suitably, then we have

$$
\mathscr{2}^{* \tau} \cong \hat{\mathscr{Q}}^{*}
$$

Let $u$ be a point of $\mathscr{S}^{r}$ such that $\mathscr{2}_{y}^{\tau}$ is isomorphic to $\hat{\mathscr{Q}}_{u}=\left(\hat{A}_{u}, \hat{\mathscr{C}}_{u}, \hat{\theta}_{u}\right)$. There is an anti-isomorphism $\theta_{u}^{*}$ of $Z$ into $\operatorname{End}_{\mathbf{Q}}\left(\hat{A}_{u}\right)$ which extends $\hat{\theta}_{u}$ and such that $\left(\hat{A}_{u}, \hat{\mathscr{C}}_{u}, \hat{\theta}_{u}^{*}\right) \cong \mathcal{Q}^{* \tau}$. Then $\hat{\theta}_{u}^{*}$ defines a $K$-linear embedding $f^{*}$ of $K \otimes_{F} Y$ into $M_{n}(L)$. 
Regard $L^{n}$ as a $Z$-module by setting $x(a \otimes b)=a x\left(f^{*}(b)\right)$. Then $\hat{\mathscr{Q}}_{u}^{*}=\left(\hat{A}_{u}, \hat{\mathscr{G}}_{u}, \hat{\theta}_{u}^{*}\right)$ is of type $\left(Z, \Xi^{\tau}\right)$. Since $\hat{\mathscr{Q}}^{*}$ is also of type $\left(Z, \Xi^{\tau}\right)$, there is an isogeny $\lambda$ of $\hat{\mathscr{Q}}^{*}$ to $\hat{\mathscr{Q}}_{u}^{*}$. Recall that for every $\alpha \in G\left(T_{1}\right)_{\mathbf{R}+}$ and $w \in \mathscr{S}^{r}$ there is a C-linear automorphism $\Lambda_{1}(\alpha, w)$ of $\mathrm{C}^{4 n g}$ such that

$$
\Lambda_{1}(\alpha, w) \mathfrak{y}_{1}(x, w)=\mathfrak{y}_{1}\left(x \alpha, \alpha^{-1}(w)\right)
$$

for all $x \in L_{\mathbf{R}}^{n}$, see $[8,4.4]$. The isogeny $\lambda$ is induced by $\Lambda_{1}\left(\beta, y_{1}\right)$ for some $\beta \in G\left(T_{1}\right)_{\mathbf{Q}}$ such that $\beta(u)=y_{1}$. Let $\xi: \mathbf{C}^{4 n g} \rightarrow \hat{A}_{u}$ be a surjective holomorphic homomorphism with $\mathfrak{y}_{1}\left(\mathfrak{M}_{1}, u\right)$ as kernel. Then from the definitions of $\hat{\theta}^{*}$ and $\hat{\theta}_{u}^{*}$ we have

$$
\xi\left(\mathfrak{y}_{1}\left(x i_{1}\left(f_{1}(b)\right) \beta, u\right)\right)=\xi\left(\mathfrak{y}_{1}\left(x \beta f^{*}(b)\right), u\right)
$$

for all $x \in L_{\mathbf{R}}^{n}$ and $b \in Y$.

Consider the PEL-type $\Omega^{\prime}=\left(L, \Phi_{1}, \rho ; \mathfrak{M}_{1} \beta^{-1}, v(\beta) T_{1}\right)$ and let $\mathfrak{y}_{1}^{\prime}(x, w)$ $=\mathfrak{y}_{1}\left(x \beta, \beta^{-1}(w)\right)$. Let $\Sigma\left(\Omega^{\prime}\right)=\left\{\mathscr{Q}_{w}^{\prime} \mid w \in \mathscr{S}^{r}\right\}$ be the family of PEL-structures of type $\Omega^{\prime}$ parametrized by $\mathfrak{y}_{1}^{\prime}$. We have $\mathscr{Q}_{w}^{\prime} \cong \widehat{\mathscr{V}}_{v}$, where $v=\beta^{-1}(w)$. Note that $\Lambda_{1}^{\prime}(\alpha, w)$ $=\Lambda_{1}\left(\beta^{-1} \alpha \beta, \beta^{-1}(w)\right)$ has the property

$$
\Lambda_{1}^{\prime}(\alpha, w) \mathfrak{y}_{1}^{\prime}(x, w)=\mathfrak{y}_{1}^{\prime}\left(x \alpha, \alpha^{-1}(w)\right)
$$

for all $x \in L_{\mathbf{R}}^{n}$. Extend $\mathscr{Q}_{y_{1}}^{\prime}$ to a structure $\mathscr{Q}^{\prime *}$ with the help of $\left(Y, P, \delta, f_{1}\right)$. Then it follows from (2.7.2) that $\mathscr{2}^{*} \cong \hat{\mathscr{Q}}_{u}^{*} \cong \mathscr{Q}^{* \tau}$.

Now replace $\mathfrak{M}_{1}$ by $\mathfrak{M}_{1} \beta^{-1}, T_{1}$ by $v(\beta) T_{1}$ and $\Omega^{\prime}$ by $\hat{\Omega}$. Replace $\mathfrak{y}_{1}$ by $\mathfrak{y}_{1}^{\prime}$, and let $\Sigma(\hat{\Omega})=\left\{\widehat{\mathscr{Q}}_{w} \mid w \in \mathscr{S}^{r}\right\}$ stand for the family parametrized by the new $\mathfrak{y}_{1}$. Then the above reasoning shows that $\mathscr{Q}^{* \tau} \cong \hat{\mathscr{Q}}^{*}$. Especially $\mathscr{Q}_{y}^{\tau}$ is isomorphic to $\hat{\mathscr{Q}}_{y_{1}}$.

2.8. Now apply the results of $[6 \mathrm{II}, \S 3]$ to $\mathscr{2}^{*}=\left(A_{y}, \mathscr{C}_{y}, \theta^{*}\right)$ and $\tau$. (We emphasize one more time that the results hold for any $\tau$.) For every finite prime $\not h$ of $F$, there are a $Z_{h_{h}}$-linear automorphism $\alpha_{\not h}$ of $L_{\not h}^{n}$ and $c_{k h} \in \mathbf{Q}_{p}^{\times}(\not \mid p)$ such that $\mathfrak{M}_{k} \alpha_{k}=\mathfrak{M}_{1 \not k}$ and $U(x, y)=c_{n} U_{1}\left(x \alpha_{n}, y \alpha_{n}\right)$ for all $x, y \in L_{k}^{n}$. Furthermore, let $s$ be any non-negative integer and $\left(t_{1}, \ldots, t_{s}\right)\left(\right.$ resp. $\left.\left(t_{11}, \ldots, t_{1 s}\right)\right)$ be an ordered set of elements from $L^{n} / \mathfrak{M}$ (resp. $\left.L^{n} / \mathfrak{M}_{1}\right)$. Then we have $t_{1 i} \equiv t_{i} \alpha_{\mu}\left(\bmod \mathfrak{M}_{1 \not}\right)$ for all $i=1, \ldots, s$, provided the conjugation of $\left(Z, \Xi, \mathfrak{M}, U ; t_{1}, \ldots, t_{s}\right)$ under $\tau$ is $\left(Z, \Xi_{1}, \mathfrak{M}_{1}, U_{1} ; t_{11}, \ldots, t_{1 s}\right)$. From the relation between $U$ and $U_{1}$, we obtain $T=c_{k} \alpha_{k} T_{1} \cdot{ }^{t} \alpha_{k}^{\rho}$. Observe that $\alpha_{k}$ is a $Z_{k}$-automorphism means it is an $L_{k}$-automorphism and

$$
\alpha_{p}^{-1} i(f(a)) \alpha_{p h}=i_{1}\left(f_{1}(a)\right) \quad \text { for all } a \in Y_{p h} \text {. }
$$

Let $S$ and $S_{1}$ be as in 2.1. Then we have

$$
(i(f(a)))^{\iota \rho} S=S i(f(a)), \quad\left(i_{1}\left(f_{1}(a)\right)\right)^{\iota \rho} S_{1}=S_{1} i_{1}\left(f_{1}(a)\right)
$$

for all $a \in Y_{n}$. On the other hand, it follows from (2.8.1) that

$$
\left(i_{1}\left(f_{1}(a)\right)\right)^{\imath \rho}\left[\left(\alpha_{h}^{-1}\right)^{\imath \rho} S \alpha_{h}\right]=\left[\left(\alpha_{h}^{-1}\right)^{i \rho} S \alpha_{h}\right]\left(i_{1}\left(f_{1}(a)\right)\right) .
$$

Hence $S_{1}^{-1}\left(\alpha_{n}^{-1}\right)^{\imath \rho} S \alpha_{h}$ commutes with every element of $i_{1}\left(f_{1}\left(Y_{p}\right)\right)$, i.e. it belongs to the commutor of $i_{1}\left(f_{1}\left(Y_{h}\right)\right)$ in $M_{n}\left(L_{k}\right)$, which is $K_{h}\left(i_{1}\left(f_{1}\left(P_{f}\right)\right)\right)$. Therefore there are $a \in K_{p}$ and $\lambda \in P_{p}$ such that

$$
\left(\alpha_{p}^{-1}\right)^{\imath \rho} S \alpha_{\mu}=a S_{1} i_{1}\left(f_{1}(\lambda)\right)
$$


We show that $\lambda$ is in $F_{p_{k}}$.

Combining $T=c_{p} \alpha_{k} T_{1} \cdot{ }^{t} \alpha_{k}^{\rho}$ with (2.8.2), we have

$T \cdot{ }^{t} S^{\rho}=c_{\not p} a^{\rho} \alpha_{p h} T_{1} \cdot{ }^{t}\left(i_{1}\left(f_{1}(\lambda)\right)\right)^{\rho} \cdot{ }^{t} S_{1}^{\rho} \cdot{ }^{t} \alpha_{\not h}^{t}$,

and

$$
-S^{\imath \rho} T^{i \rho}=-c_{h} a^{\rho} \alpha_{p h} S_{1}^{\iota \rho}\left(i_{1}\left(f_{1}(\lambda)\right)\right)^{\imath \rho} T_{1}^{i \rho} \cdot{ }^{t} \alpha_{p}^{2} .
$$

Since $T \cdot{ }^{t} S^{\rho}=-S^{i \rho} T^{i \rho}, T_{1} \cdot{ }^{t} S_{1}^{\rho}=-S_{1}^{i \rho} T_{1}^{i \rho}($ see (2.1.1)), this shows

$$
T_{1} \cdot{ }^{t}\left(i_{1}\left(f_{1}(\lambda)\right)\right)^{\rho} \cdot{ }^{t} S_{1}^{\rho}=-S_{1}^{i \rho}\left(i_{1}\left(f_{1}(\lambda)\right)\right)^{\imath \rho} T_{1}^{\imath \rho} .
$$

Because $f_{1}(\lambda) \in M_{n}\left(B_{1 / n}\right)$, we have $S_{1}\left(i_{1}\left(f_{1}(\lambda)\right)\right)=\left(i_{1}\left(f_{1}(\lambda)\right)\right)^{\imath \rho} S_{1}$. So the left hand side of (2.8.3) is equal to

$$
T_{1}^{i \rho} \cdot{ }^{t}\left(i_{1}\left(f_{1}(\lambda)\right)\right)^{i}=\left(i_{1}\left(f_{1}(\lambda)\right)\right)^{\imath \rho} T_{1}^{i \rho} .
$$

Applying $\imath \rho$ to the identity, we obtain

$$
T_{1} \cdot{ }^{t}\left(i_{1}\left(f_{1}(\lambda)\right)\right)^{\rho}=i_{1}\left(f_{1}(\lambda)\right) T_{1} .
$$

On the other hand,

$$
T_{1} \cdot{ }^{t}\left(i_{1}\left(f_{1}(\lambda)\right)\right)^{\rho} T_{1}^{-1}=i_{1}\left({ }^{t} f_{1}(\lambda)^{2}\right)=i_{1}\left(f_{1}\left(\lambda^{\delta}\right)\right) .
$$

Therefore $\lambda=\lambda^{\delta}$, and hence $\lambda \in F_{p}$. This proves:

$$
\left(\alpha_{p}^{-1}\right)^{\imath \rho} S \alpha_{h}=d_{p h} S_{1} \quad \text { for some } d_{p} \in K_{p} .
$$

Note that

(2.8.5) $d_{n} d_{p}^{\rho}=\kappa / \kappa_{1}$.

This results from (2.8.4) and the identities $S^{\iota \rho} S=\kappa 1_{n}, S_{1}^{\iota \rho} S_{1}=\kappa_{1} 1_{n}$.

2.9. Define an isomorphism $\mathfrak{u}_{k}$ of $G_{k}^{*}$ onto $G_{1 \not k}^{*}$ by $\mathfrak{u}_{k}(\alpha)=\alpha_{k}^{-1} \alpha \alpha_{k}$. Then (2.8.4) shows that $\mathfrak{u}_{k}$ actually sends $G_{\not h}$ isomorphically onto $G_{1 \not k}$. Since $\mathfrak{M}_{\not k}=\mathfrak{M}_{1 \not k}$ for almost all $\not, \mathfrak{u}_{n}$ 's can be put together to form an isomorphism $\mathfrak{u}_{0}$ of $G_{0}$ to $G_{10}$. For an infinite prime $\not_{\lambda}$ corresponding to the embedding $\tau_{\lambda}$, the signature of $T$ at $\not_{\lambda}$ is the same as that of $T_{1}$ at $\not_{\mu}$ if $\tau_{\lambda} \tau=\tau_{\mu}$. Therefore we can define an isomorphism $\mathfrak{u}_{\lambda}$ of $G_{k_{\lambda}}^{*}$ onto $G_{1 k_{\mu}}^{*}$ which sends $G_{h_{\lambda}}$ onto $G_{1 k_{\mu}}$. Putting together all $\mathfrak{u}_{\lambda}$ 's we get an isomorphism $\mathfrak{u}_{\infty}$ of $G_{\mathbf{R}_{+}}^{*}$ on to $G_{1 \mathbf{R}+}^{*}$ which sends $G_{\mathbf{R}_{+}}$onto $G_{1 \mathbf{R}_{+}}$. Finally by putting $\mathfrak{u}_{0}$ and $\mathfrak{u}_{\infty}$ together, we obtain an isomorphism $\mathfrak{u}$ of $G_{\mathbf{A}_{+}}^{*}$ onto $G_{1 \mathbf{A}}^{*}$ that sends $G_{\mathbf{A}_{+}}$onto $G_{1 \mathrm{~A}+}$. By Lemma 1.5 , we have $\mathfrak{u}\left(\overline{\mathscr{G}}_{+}\right)=\overline{\mathscr{G}}_{1+}$. Hence $\mathfrak{u}$ induces an isomorphism (again denoted by $\mathfrak{u}$ ) of $\mathfrak{U}^{1}$ onto $\mathfrak{U}_{1}^{1}$.

2.10. Now it is only a formality to extend $\mathfrak{u}$ to an isomorphism of $\mathfrak{A}$ onto $\mathfrak{A}_{1}$. First observe that both $\mathfrak{X} / \mathfrak{U}^{1}$ and $\mathfrak{U}_{1} / \mathfrak{U}_{1}^{1}$ are canonically isomorphic to the finite group $\mathrm{g}$ defined in [CII, 4.1]. Let the notation be as in Proposition 4.6 of [CII] and its proof. Write

$$
A_{+}=\bigcup_{\alpha \in U} \alpha A_{+}^{1} \text { and } A_{1+}=\bigcup_{\alpha_{1} \in U_{1}} \alpha_{1} A_{1+}^{1} .
$$


For $\alpha \in U$ put $\mathfrak{u}(\alpha)=\alpha_{1} \in U_{1}$ if $\alpha$ and $\alpha_{1}$ have the same image in $\mathfrak{g}$. Define $c_{\alpha}$ $=\psi_{1}(\mathfrak{u}(\alpha))^{-1} \mathfrak{H}(\psi(\alpha)) \in \mathfrak{A}_{1}^{1}$, and for $(\alpha, x) \in U \times \mathfrak{A}^{1}=\mathfrak{A}$ set

$$
\mathfrak{u}(\alpha, x)=\left(\mathfrak{u}(\alpha), c_{\alpha} \mathfrak{u}(x)\right) \in U_{1} \times \mathfrak{U}_{1}^{1}=\mathfrak{U}_{1} .
$$

Then a trivial computation shows that $\mathfrak{u}$ is an isomorphism of $\mathfrak{A}$ onto $\mathfrak{A}_{1}$. In $\S 3$ we shall prove that $\mathfrak{u}$ satisfies the conditions of Theorem 1.3.

\section{Proof of the Main Theorem}

3.1. Proposition. Let $\mathfrak{M}_{1}$ be determined as in 2.7. Then there are an ample $\mathfrak{r}_{F}$-lattice $\mathrm{m}_{1}$ in $B_{1}^{n}$ and an element $f$ of $L_{\mathbf{A}}^{\times}$such that $\mathfrak{M}_{1}=f \mathrm{r}_{K} \cdot i_{1}^{\prime}\left(\mathrm{m}_{1}\right)$.

Proof. Let $v$ and $v_{1}$ be as in 2.1. As we saw in the proof of Proposition 2.2, for every finite prime $\not k$ of $F$, there are $c_{k} \in F_{k}^{\times}$and $b_{k} \in L_{k}^{\times}$such that $v_{1}=c_{k} b_{k} v b_{k}^{p}$. For $a_{k}$ $=c_{h} N\left(b_{k}^{\rho}\right) \in K_{k}^{\times}$, we have $a_{h} a_{k}^{\rho}=\kappa / \kappa_{1}$, see Proposition 2.2. On the other hand, by

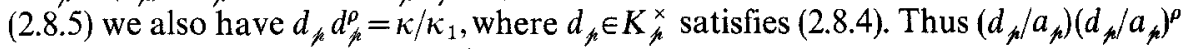
$=1$. Hence there is $e_{k} \in K_{n}^{\times}$such that

(3.1.1) $d_{p h} / a_{p}=e_{p h}^{\rho} / e_{p h}$.

Put $f_{h}=e_{\not_{k}} b_{k} \in L_{p^{x}}^{\times}$. For an infinite prime $\not h$, let $f_{k}$ be an arbitrary element of $L_{k}^{\times}$. It is easy to see that $f=\left(f_{n}\right)$ belongs to $L_{\mathbf{A}}^{\times}$. Define $m_{1}$ by

$$
i_{1}^{\prime}\left(m_{1}\right)=f^{-1} \mathfrak{M}_{1} \cap i_{1}^{\prime}\left(B_{1}^{n}\right) \text {. }
$$

We show that for every finite $\not$,

(3.1.2) $\quad i_{1}^{\prime}\left(B_{1 p h}^{n}\right)=f_{k} \cdot i^{\prime}\left(B_{p h}^{n}\right) \alpha_{p h}$.

In fact, we have

$$
\begin{aligned}
i^{\prime}\left(B^{n}\right) & =\left\{x \in L^{n} \mid x=v x^{i \rho} S\right\}, \\
i_{1}^{\prime}\left(B_{1}^{n}\right) & =\left\{x \in L^{n} \mid x=v_{1} x^{i \rho} S_{1}\right\} .
\end{aligned}
$$

Therefore, for $x_{h} \in B_{\mu}^{n}$ we have

$$
\begin{aligned}
& v_{1}\left(f_{h} \cdot i^{\prime}\left(x_{p h}\right) \alpha_{h}\right)^{i \rho} S_{1}=v_{1} e_{h}^{\rho} b_{h}^{i \rho} \cdot i^{\prime}\left(x_{h}\right)^{i \rho} \alpha_{h}^{\imath \rho} S_{1}
\end{aligned}
$$

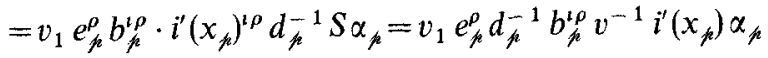

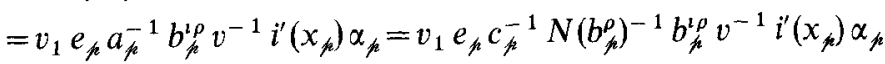

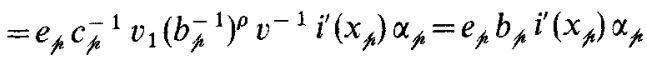

$$
\begin{aligned}
& =f_{n} \cdot i^{\prime}\left(x_{n}\right) \alpha_{n} \text {. }
\end{aligned}
$$

This completes the proof of (3.1.2). Especially we have

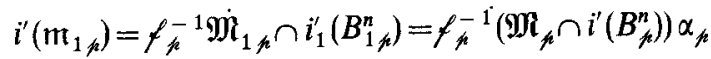

$$
\begin{aligned}
& =f_{p h}^{-1} \cdot i^{\prime}\left(\mathbf{m}_{p}\right) \alpha_{k} .
\end{aligned}
$$




\section{Hence}

$$
\mathfrak{r}_{K_{k}} \cdot i_{1}^{\prime}\left(\mathfrak{m}_{1 \not h}\right)=f_{k}^{-1} \mathfrak{M}_{k} \alpha_{k}=f_{k}^{-1} \mathfrak{M}_{1 \not h} .
$$

This being true for all finite primes $\not$, we have $\mathfrak{r}_{K} \cdot i_{1}^{\prime}\left(\mathfrak{m}_{1}\right)=f^{-1} \mathfrak{M}_{1}$. In view of (3.1.3), $m_{1}$ is ample because $m$ is.

\subsection{Put}

$$
\begin{aligned}
& \Gamma^{*}=\left\{\gamma \in G_{\mathbf{Q}}^{*} \mid v(\gamma)=1, \mathfrak{M} \gamma=\mathfrak{M}\right\}, \\
& \Gamma_{1}^{*}=\left\{\gamma \in G_{1 \mathbf{Q}}^{*} \mid v_{1}(\gamma)=1, \mathfrak{M}_{1} \gamma=\mathfrak{M}_{1}\right\} .
\end{aligned}
$$

Let $\Omega$ and $\hat{\Omega} \sim \Omega^{\tau}$ be as in 2.7, and let $(V, v, \phi)$ be a moduli-system for $\Sigma(\Omega)$ in the sense of $[7,6.2]$. Here $v$ is an assignment which assigns a point $v(2)$ of $V$ to every PEL-structure $\mathscr{Q}$ of type $\Omega, \phi$ is a holomorphic map from $\mathscr{S}^{r}$ onto $V$ inducing a biregular isomorphism of $\mathscr{S}^{r} / \Gamma^{*}$ onto $V$, and $\phi(w)=v\left(\mathscr{Q}_{w}\right)$ for all $w \in \mathscr{S}^{r}$. Let $\left(V_{1}, v_{1}, \phi_{1}\right)$ be a moduli-system for $\Sigma(\hat{\Omega})$. Since $\hat{\Omega} \sim \Omega^{\tau}$, by $[8,4.23]$ there is a biregular isomorphism $\psi$ of $V_{1}$ to $V^{\tau}$ such that $v^{\tau}=\psi \circ v_{1}$.

3.3. Proposition. Let $z, z_{1}, y=j(z)$ and $y_{1}=j_{1}\left(z_{1}\right)$ be the special points chosen in 2.6. Let $\beta \in G_{\mathbf{Q}+}$ and $w=j(\beta(z))=i(\beta)(y)$. Then there is $\gamma \in G_{1 \mathbf{Q}+}$ such that for $w_{1}=j_{1}\left(\gamma\left(z_{1}\right)\right)$ we have $\phi(w)^{x}=\psi\left(\phi_{1}\left(w_{1}\right)\right)$.

Proof. Let $\beta_{1}=\mathfrak{u}(\beta) \in G_{1 \mathrm{~A}+}$. Then $v_{1}\left(\beta_{1}\right)=v(\beta) \in F^{\times}$. Hence by [A, 3.3], there is $\alpha \in G_{1 Q+}$ such that $v_{1}(\alpha)=v_{1}\left(\beta_{1}\right)$. Put $S_{1}=\left\{x \in \overline{\mathscr{G}}_{1+} \mid \mathrm{m}_{1} x=\mathrm{m}_{1}\right\}$. Then $S_{1} \in \mathcal{Z}_{1}$. By the strong approximation theorem for $G_{1}^{u}$ (see [CI, 3.4]), there are $\alpha^{\prime} \in G_{1 Q}^{u}$ and $s \in S_{1} \cap G_{1 \mathrm{~A}}^{u}$ such that $\beta_{1} \alpha^{-1}=s \alpha^{\prime}$. Put $\gamma=\alpha^{\prime} \alpha \in G_{1 \mathrm{Q}+}$. Then $v_{1}(\gamma)=v_{1}(\alpha)=v(\beta)$. We show that $\gamma$ satisfies the condition of our proposition.

As in 2.7 , we first consider $L^{n}$ as a $Z$-module by setting $x(a \otimes b)=a x(i(f(b)))$. The extended structure $\mathscr{2}^{*}$ of $\mathscr{2}_{y}$ is then of type $(Z, \Xi, \mathfrak{M}, U)$. We see easily that $\mathscr{Q}_{w}$ can be extended to a structure $\mathscr{2}_{w}^{*}$ of type $\left(Z, \Xi, \mathfrak{M} \beta, U_{\beta}\right)$, where $U_{\beta}\left(a, a^{\prime}\right)$ $=\operatorname{tr}_{L / \mathbf{Q}}\left(v(\beta)^{-1} T\left(a, a^{\prime}\right)\right)$. Now regard $L^{n}$ as a $Z$-module by setting $x(a \otimes b)$ $=\operatorname{ax}\left(i_{1}\left(f_{1}(b)\right)\right)$ instead. Then $\widehat{\mathscr{Q}}_{y_{1}}$ can be extended to a structure $\hat{\mathscr{Q}}^{*}$ of type $\left(Z, \Xi_{1}, \mathfrak{M}_{1}, U_{1}\right)$. Recall that we have $\mathscr{2}^{* \tau} \cong \hat{\mathscr{Q}}^{*}$, see 2.7 . We determine the type $\left(Z, \Xi_{1}, \mathfrak{M}^{*}, U^{*}\right)$ of $\mathscr{Q}_{w}^{* \tau}$.

We have $(Z, \Xi, \mathfrak{M}, U)^{\tau} \sim\left(Z, \Xi_{1}, \mathfrak{M}_{1}, U_{1}\right)$ and $\left(Z, \Xi, \mathfrak{M} \beta, U_{\beta}\right)^{\tau} \sim\left(Z, \Xi_{1}, \mathfrak{M}^{*}, U^{*}\right)$. Let $\not h$ be a finite prime of $F$. Then $\alpha_{h}$ is a $Z_{k h}$-linear automorphism of $L_{k h}^{n}$ such that

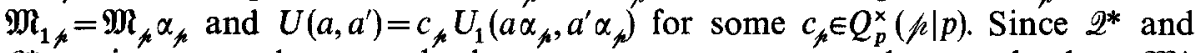
$\mathscr{Q}_{w}^{*}$ are isogenous, by a standard argument we can assume that we also have $\mathfrak{M}_{k}^{*}$

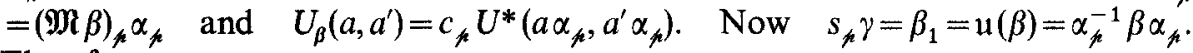
Therefore

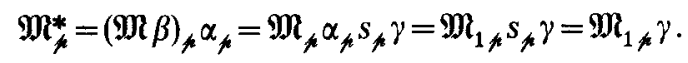

This is true for all finite $\not$. Hence $\mathfrak{M}^{*}=\mathfrak{M}_{1} \gamma$. Similarly, we have $U^{*}=U_{1 \gamma}$, where $U_{1 \gamma}\left(a, a^{\prime}\right)=\operatorname{tr}_{L / \mathbf{Q}}\left(v_{1}(\gamma)^{-1} T_{1}\left(a, a^{\prime}\right)\right)$.

So $\mathscr{2}_{w}^{* t}$ is of type $\left(Z, \Xi_{1}, \mathfrak{M}_{1} \gamma, U_{1 y}\right)$, which is the type of the extended structure of $\hat{\mathscr{Q}}_{w_{1}}$, where $w_{1}=j_{1}\left(\gamma\left(z_{1}\right)\right)$. Hence $\mathscr{Q}_{w}^{\tau} \cong \widehat{\mathscr{Q}}_{w_{1}}$. Therefore, $v\left(\mathscr{Q}_{w}\right)^{\tau}=\psi\left(v_{1}\left(\mathscr{Q}_{w}^{\tau}\right)\right)$ $=\psi\left(v_{1}\left(\widehat{Q}_{w_{1}}\right)\right)$, or $\phi(w)^{\tau}=\psi\left(\phi_{1}\left(w_{1}\right)\right)$. 
3.4. For a positive integer $b$, define a member $S(\mathrm{~m}, b)\left(\operatorname{resp} . S_{1}\left(\mathrm{~m}_{1}, b\right)\right)$ of $\mathscr{Z}\left(\right.$ resp. $\left.\mathscr{Z}_{1}\right)$ as in [CI, 2.10]. Then it follows from Proposition 3.1 that $\mathfrak{u}(S(\mathfrak{m}, b))=S_{1}\left(\mathfrak{m}_{1}, b\right)$. For any $\mathfrak{r}_{K}$-lattice $\mathfrak{N}$ in $L^{n}$, and any positive integer $b$, put

$$
\begin{aligned}
& \Gamma^{*}(\mathfrak{N}, b)=\left\{\gamma \in G_{\mathbf{Q}}^{*} \mid v(\gamma)=1, \mathfrak{N} \gamma=\mathfrak{N}, \mathfrak{N}(1-\gamma) \subset b \mathfrak{N}\right\} \\
& \Gamma_{1}^{*}(\mathfrak{N}, b)=\left\{\gamma \in G_{1 \mathbf{Q}}^{*} \mid v_{1}(\gamma)=1, \mathfrak{N} \gamma=\mathfrak{N}, \mathfrak{N}(1-\gamma) \subset b \mathfrak{N}\right\} .
\end{aligned}
$$

For a given positive integer $a$, choose $b$ and $c$ so that conditions (6.4.2)-(6.4.4) of $[\mathrm{CI}]$ are satisfied. Put

$$
S=S(m, c) \cdot\{x \in S(m, b) \mid v(x)=1\} .
$$

Then

$$
\mathfrak{u}(S)=S_{1}=S_{1}\left(\mathrm{~m}_{1}, c\right) \cdot\left\{x \in S_{1}\left(\mathrm{~m}_{1}, b\right) \mid v_{1}(x)=1\right\} .
$$

We assume that $b$ has been chosen in such a way that the following conditions corresponding to [CI, (6.4.3), (6.4.4)] also hold:

(3.4.3) Put $E=\mathfrak{r}_{F}^{\times}$. Then, for every $u_{1} \in G_{1_{\mathbf{A}}}$,

$$
E \cdot \Gamma\left(u_{1}^{-1} S_{1} u_{1}\right)=\left\{\alpha \in E \cdot \Gamma_{1}^{*}\left(f^{-1} \mathfrak{M}_{1} u_{1}, b\right) \mid \alpha\left(\mathscr{D}_{n}^{r}\right) \cap \mathscr{D}_{n}^{r} \neq \emptyset\right\} .
$$

(3.4.4) For every $u_{1} \in G_{1 \mathrm{~A}}, \Gamma_{1}^{*}\left(f^{-1} \mathfrak{M}_{1} u_{1}, b\right)$ has no element of finite order other than the identity element.

Note that

$$
\Gamma_{1}^{*}\left(f^{-1} \mathfrak{M}_{1} u_{1}, b\right)=\Gamma_{1}^{*}\left(\mathfrak{M}_{1} u_{1}, b\right)
$$

Let $\left(H, \Phi^{\prime}\right)$ (resp. $\left.\left(H_{1}, \Phi_{1}^{\prime}\right)\right)$ be the reflex of $(K, \Phi)$ (resp. $\left.\left(K, \Phi_{1}\right)\right)$. Then by Lemma 1.5, $H_{1}=H^{\tau}$. Let $M_{c}$ be the class field over $H$ corresponding to the subgroup $H^{\times} \cdot\left\{h \in H_{\mathbf{A}}^{\times} \mid h \equiv 1 \bmod _{0}(c)\right\}$. Define a class field $M_{1_{c}}$ over $H_{1}$ in the same way. Then $M_{1 c}=M_{c}^{\tau}$.

3.5. Choose $q_{1}, \ldots, q_{s} \in L^{n} / \mathfrak{M}$ such that $b^{-1} \mathfrak{M} / \mathfrak{M} \cong \sum_{i=1}^{s} \mathbf{Z} q_{i}$. Consider the PELtype

$$
\Omega^{\prime}=\left(L, \Phi, \rho ; \mathfrak{M}, T ; q_{1}, \ldots, q_{s}\right) .
$$

Fix $q_{11}, \ldots, q_{1 s} \in L^{n} / \mathfrak{M}_{1}$ so that

$$
\hat{\Omega}^{\prime}=\left(L, \Phi_{1}, \rho ; \mathfrak{M}_{1}, T_{1} ; q_{11}, \ldots, q_{1 s}\right)
$$

is equivalent to $\Omega^{\prime \tau}$. Then for every finite prime $\not h$ of $F$, we have $\mathfrak{M}_{1 \not h}=\mathfrak{M}_{\not} \boldsymbol{\alpha}_{\not}$ and $q_{1 i} \equiv q_{i} \alpha_{h}\left(\bmod \mathfrak{M}_{1 \beta^{\prime}}\right)$. Therefore $b^{-1} \mathfrak{M}_{1} / \mathfrak{M}_{1} \cong \sum_{i=1}^{s} \mathbf{Z} q_{1 i}$. Let $k_{\Omega^{\prime}}$ be the field of moduli of $\Omega^{\prime}$. Then $k_{\Omega^{\prime}}$ is contained in $M_{\mathrm{c}}$ [CI. (6.6.1)]. The field of moduli of $\hat{\Omega}^{\prime}$ is $k_{\Omega^{\prime}}^{\tau}$, which is contained in $M_{1 \mathrm{c}}$.

Let $\mathfrak{y}(x, w)\left(\right.$ resp. $\left.\mathfrak{y}_{1}(x, w)\right)$ be the parametrizing function for $\Sigma(\Omega)($ resp. $\Sigma(\hat{\Omega}))$ as given in 2.7 . We can use the same $\mathfrak{y}(x, w)\left(\right.$ resp. $\left.\mathfrak{y}_{1}(x, w)\right)$ to parametrize a family 
$\Sigma\left(\Omega^{\prime}\right)=\left\{\mathscr{Q}_{w}^{\prime} \mid w \in \mathscr{S}^{r}\right\} \quad$ (resp. $\left.\Sigma\left(\hat{\Omega}^{\prime}\right)=\left\{\hat{\mathscr{Q}}_{w}^{\prime} \mid w \in \mathscr{S}^{r}\right\}\right)$ of PEL-structures of type $\Omega^{\prime}$ (resp. $\left.\hat{\Omega}^{\prime}\right)$. Let $\left(V^{\prime}, u^{\prime}, \phi^{\prime}\right)$ (resp. $\left.\left(V_{1}, v_{1}, \phi_{1}\right)\right)$ be a moduli-system for $\Sigma\left(\Omega^{\prime}\right)$ (resp. $\Sigma\left(\hat{\Omega}^{\prime}\right)$ ). We can identify $V_{S}$ with the subvariety $\phi^{\prime}\left(\mathscr{D}_{n}^{r}\right)$ of $V^{\prime}$ and take $\phi_{S}=\phi^{\prime} \circ j$ [CI. 6.9]. Similarly, in view of (3.4.3)-(3.4.5), we can identify $V_{S_{1}}$ with $\phi_{1}\left(\mathscr{D}_{n}^{r}\right)$ and take $\phi_{S_{1}}$ $=\phi_{1}^{\prime} \circ j_{1}$.

Since $\hat{\Omega}^{\prime} \sim \Omega^{\prime \tau}$, there is a biregular isomorphism $\psi_{\Omega}$ of $V_{1}^{\prime}$ to $V^{\prime \tau}$ over $M_{1 \mathfrak{c}}$ such that $v^{\prime \tau}=\psi \circ v_{1}^{\prime}$. We show that $\psi_{\Omega}$ restricted to $V_{S_{1}}$ defines an isomorphism of $V_{S_{1}}$ to $V_{S}^{\tau}$ (over $M_{1 c}$ ). Let $z \in \mathscr{H}$ be as in 3.3. Then $Z=\left\{\beta(z) \mid \beta \in G_{\mathbf{Q}+}\right\}$ is dense in $\mathscr{H}$. So it is sufficient to show that $\left(\phi^{\prime}(j(Z))\right)^{\tau}$ is contained in $\psi_{\Omega}\left(V_{S_{1}}\right)$, and is Zariski dense in there.

Let $\beta \in G_{\mathbf{Q}^{+}}$and $w=j(\beta(z))$. Let the notation be as in 3.2. Then Proposition 3.3 shows that $\phi(w)^{\tau}=\psi_{\Omega}\left(\phi_{1}\left(w_{1}\right)\right)$ for some $w_{1} \in \mathscr{D}_{n}^{r}$. According to [9], $\mathscr{2}^{\tau} \cong \hat{\mathscr{Q}}_{w_{1}}$ posesses a certain non-holomorphic endomorphism $D$. It follows that $\mathscr{2}_{w}^{\prime \tau}$ also posesses this endomorphism $D$. Therefore $\mathscr{Q}_{w}^{\prime \text { t }}$ is isomorphic to $\hat{\mathscr{Q}}_{v_{1}}^{\prime}$ for some $v_{1} \in \mathscr{D}_{n}^{r}$. Thus $\phi^{\prime}(w)^{\tau}=\psi_{\Omega}\left(\phi_{1}^{\prime}\left(v_{1}\right)\right) \in \psi_{\Omega}\left(\phi_{1}^{\prime}\left(j_{1}(\mathscr{H})\right)\right)=\psi_{\Omega}\left(V_{S_{1}}\right)$. This shows $\left(\phi^{\prime}(j(Z))\right)^{\tau}$, and hence its Zariski closure $V_{S}^{\mathrm{r}}$, is contained in $\psi_{\Omega}\left(V_{S_{1}}\right)$. Similarly, we show that $\psi_{\Omega}\left(V_{S_{1}}\right)$ is contained in $V_{S}^{\tau}$. Therefore the restriction $\psi_{S}$ of $\psi_{\Omega}$ to $V_{S_{1}}$ is a biregular isomorphism of $V_{S_{1}}$ to $V_{S}^{\tau}$.

Thus for $S$ of the form (3.4.1) with sufficiently large $b$, and for $S_{1}=\mathfrak{u}(S)$, there is a biregular isomorphism $\psi_{S}$ of $V_{S_{1}}$ to $V_{S}^{\tau}$ rational over $M_{1 c}$. Put $\hat{\phi}_{S}=\psi_{S} \circ \phi_{S_{1}}$. Then $\left(V_{S}^{\tau}, \tilde{\phi}_{S}\right)$ is a model of $\mathscr{H} / \Gamma_{S_{1}}$ over $k_{S}^{\tau}$.

3.6. For $x \in G_{\mathbf{A}+}$ and $x_{1}=\mathfrak{u}(x) \in G_{1_{\mathbf{A}}+}$ we have $v_{1}\left(x_{1}\right)=v(x)$. Therefore, by the definitions of $\rho$ and $\rho_{1}$ in 1.1, and by Lemma 1.5, we have $\tau \rho_{1}\left(x_{1}\right)=\rho(x) \tau$. Similarly, if $S \in \mathscr{Z}$ and $S_{1}=u(S) \in \mathscr{Z}_{1}$, then $k_{S}^{\tau}=k_{S_{1}}$.

3.7. Let $\mathscr{G}_{+}^{*}$ be the subgroup of $G_{\mathbf{A}}^{*}$ defined in [CI, 6.2]. Define a subgroup $\mathscr{G}_{1+}^{*}$ of $G_{1 \mathrm{~A}+}^{*}$ correspondingly. Then we have $\mathfrak{u}\left(\mathscr{G}_{+}^{*}\right)=\mathscr{G}_{1+}^{*}$ in view of Lemma 1.5. Let $\mathscr{G}_{H+}$, $\lambda_{H}$, etc. be as in [CI, 3.1], and define $\mathscr{G}_{1 H_{1+}}, \lambda_{H_{1}}$ in a similar way. Let $S$ and $S_{1}$ be as in 3.4. For $x \in \mathscr{G}_{H_{+}}, x_{1}=\mathfrak{u}(x) \in \mathscr{G}_{1_{H_{1}}}$, put $T=x^{-1} S x$, and $T_{1}=x_{1}^{-1} S_{1} x_{1}=\mathfrak{u}(T)$. We show that $V_{T}^{\tau}$ and $V_{T_{1}}$ are biregularly isomorphic over $M_{1 c}$.

By [CI, (6.3.5)], there is $d \in H_{\mathrm{A}}^{\times}$such that $v(x) / \lambda_{H}(d) \in F_{+}^{\times} F_{\infty++}^{\times}$and $\pi(d) x \in \mathscr{G}_{+}^{*}$. (For the notation used here and in the following, see [CI, §6].) Let $d_{1}=d^{\tau} \in H_{1 \mathbf{A}}^{\times}$. Then $\lambda_{H_{1}}\left(d_{1}\right)=\lambda_{H}(d)$ and $\pi_{1}\left(d_{1}\right)=\pi(d)$. Hence

$$
v_{1}\left(x_{1}\right) / \lambda_{H_{1}}\left(d_{1}\right) \in F_{+}^{\times} F_{\infty+}^{\times} \text {and } \pi_{1}\left(d_{1}\right) x_{1} \in \mathscr{G}_{1+}^{*} .
$$

Let $\Omega^{\prime}, \hat{\Omega}^{\prime} \sim \Omega^{\prime \text { t }}$ be as in 3.5. Put $\sigma=\left[d^{-1}, H\right]$ and $\sigma_{1}=\tau^{-1} \sigma \tau=\left[d_{1}^{-1}, H_{1}\right]$. By $[\mathrm{CI},(6.6 .1)], \Omega^{\prime \sigma}$ is equivalent to

$$
\Omega^{\prime \prime}=\left(L, \Phi, \rho ; \pi(d) \mathfrak{M} x, \mu(\pi(d) x)^{-1} T,\left\{\pi(d) q_{i} x\right\}\right),
$$

while $\hat{\Omega}^{\prime \sigma_{1}}$ is equivalent to

$$
\hat{\Omega}^{\prime \prime}=\left(L, \Phi_{1}, \rho ; \pi(d) \mathfrak{M}_{1} x_{1}, \mu_{1}\left(\pi(d) x_{1}\right)^{-1} T_{1},\left\{\pi(d) q_{i} x_{1}\right\}\right) .
$$

We have $\Omega^{\prime \prime \tau} \sim \Omega^{\prime \sigma \tau} \sim \Omega^{\prime \tau \sigma_{1}} \sim \hat{\Omega}^{\prime \sigma_{1}} \sim \hat{\Omega}^{\prime \prime}$. Note that for every finite prime $\not h$ of $F$, we have $\left(\mathfrak{M}_{1} x_{1}\right)_{n}=(\mathfrak{M} x)_{k} \alpha_{n}$ and $\mu(\pi(d) x)^{-1} T=\mu_{1}\left(\pi(d) x_{1}\right) \alpha_{k} T_{1} \cdot{ }^{t} \alpha_{k}^{\rho}$.

Let $\Sigma\left(\Omega^{\prime \prime}\right)=\left\{\mathscr{Q}_{w}^{\prime \prime} \mid w \in \mathscr{S}^{r}\right\}$ (resp. $\left.\Sigma\left(\hat{\Omega}^{\prime \prime}\right)=\left\{\hat{\mathscr{Q}}_{w}^{\prime \prime} \mid w \in \mathscr{S}^{r}\right\}\right)$ be the family of PELstructures of type $\Omega^{\prime \prime}\left(\right.$ resp. $\left.\hat{\Omega}^{\prime \prime}\right)$ parameterized by $\mathfrak{y}(x, w)$ (resp. $\mathfrak{y}_{1}(x, w)$ ). Let 
$\left(V^{\prime \prime}, v^{\prime \prime}, \phi^{\prime \prime}\right)\left(\right.$ resp. $\left.\left(V_{1}^{\prime \prime}, v_{1}^{\prime \prime}, \phi_{1}^{\prime \prime}\right)\right)$ be a moduli-system for $\Sigma\left(\Omega^{\prime \prime}\right)$ (resp. $\left.\Sigma\left(\hat{\Omega}^{\prime \prime}\right)\right)$. Since $\Omega^{\prime \prime \tau} \sim \Omega^{\prime \prime}$, there is a biregular isomorphism $\psi^{\prime \prime}$ of $V_{1}^{\prime \prime}$ to $V^{\prime \prime \tau}$ such that $\vartheta^{\prime \prime \tau}=\psi^{\prime \prime} \circ v_{1 \prime}^{\prime \prime}$. Combining the arguments of $[\mathrm{CI}, 6.7]$ and 3.5 , we see that $\psi^{\prime \prime}$ induces a biregular isomorphism $\psi_{T}$ of $V_{T_{1}}$ to $V_{T}^{\tau}$ over $M_{1 c}$. Put $\tilde{\phi}_{T}=\psi_{T}$ ० $\phi_{T_{1}}$. Then $\left(V_{T}^{\tau}, \tilde{\phi}_{T}\right)$ is a model of $\mathscr{H} / \Gamma_{T_{1}}$ over $k_{T}^{\tau}$.

3.8. Let $S$ be as in 3.3. Consider $\mathscr{W}=\left\{x^{-1} S x \mid x \in \mathscr{G}_{H+}\right\}$. Let $T=x^{-1} S x \in \mathscr{W}$, $u \in \mathscr{G}_{H+}$ and $U=u^{-1} T u$. Then $J_{I^{\prime} T}(u)$ is defined, and is a morphism of $V_{T}$ onto $V_{U}^{\sigma}$ over $k_{T}$, where $\sigma=\rho_{H}(u)$. Let

$$
\Omega^{\prime}=\left(L, \Phi, \rho ; \mathfrak{M}, T ;\left\{q_{i}\right\}\right)
$$

be as in 3.5. As observed in 3.7, there is a PEL-type $\Omega^{\prime \prime}$ of the form

$$
\Omega^{\prime \prime}=\left(L, \Phi, \rho ; k \mathfrak{M} x, \kappa T,\left\{k q_{i} x\right\}\right) \quad\left(k \in K_{\mathbf{A}}^{\times}, \kappa \in F_{+}^{\times}\right)
$$

such that if $\left(V^{\prime \prime}, v^{\prime \prime}, \phi^{\prime \prime}\right)$ is a moduli-system for $\Sigma\left(\Omega^{\prime \prime}\right)$, then $V_{T}$ can be embedded in $V^{\prime \prime}$ in such a way that $\phi_{T}=\phi^{\prime \prime} \circ j$. Let $d \in H_{A}^{\times}$be such that $v(u) / \lambda_{H}(d) \in F_{+}^{\times} F_{\infty+}^{\times}$. Then $\Omega^{\prime \prime \sigma}$ is equivalent to

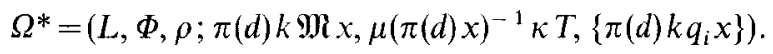

Let $\left(V^{*}, v^{*}, \phi^{*}\right)$ be a moduli-system for $\Sigma\left(\Omega^{*}\right)$. Then $V_{U}$ can be embedded in $V^{*}$ in such a way that $\phi_{U}=\phi^{*} \circ j$. Since $\Omega^{\prime \prime \sigma} \sim \Omega^{*}$, there is a biregular isomorphism $J$ from $V^{*}$ to $V^{\prime \prime \sigma}$ rational over $M_{c}$ such that $v^{\prime \prime \sigma}=J \circ v^{*}$. In view of the construction [CI, $\S 6]$, we can identify $J_{U T}(u)$ with the restriction of $J$ to $V_{T}$.

Put $x_{1}=\mathfrak{u}(x), T_{1}=x_{1}^{-1} S_{1} x_{1}, u_{1}=\mathfrak{u}(u)$ and $U_{1}=\mathfrak{u}(U)$. Also let $\sigma_{1}=\rho_{H_{1}}\left(u_{1}\right)$. Then $U_{1}=u_{1}^{-1} T_{1} u_{1}$ and $\sigma_{1}=\tau^{-1} \sigma \tau$. Let

$$
\hat{\Omega}^{\prime}=\left(L, \Phi_{1}, \rho ; \mathfrak{M}_{1}, T_{1},\left\{q_{1 i}\right\}\right)
$$

be as in 3.5. From 3.7 we know that $\Omega^{\prime \prime \tau}$ (resp. $\Omega^{* \tau}$ ) is equivalent to

$$
\begin{aligned}
& \hat{\Omega}^{\prime \prime}=\left(L, \Phi_{1}, \rho ; k \mathfrak{M}_{1} x_{1}, \kappa_{1} T_{1},\left\{k q_{1 i} x_{1}\right\}\right) \\
& \text { (resp. } \left.\hat{\Omega}^{*}=\left(L, \Phi_{1}, \rho ; \pi(d) k \mathfrak{M}_{1} x_{1}, \mu_{1}\left(\pi(d) x_{1}\right)^{-1} \kappa_{1} T_{1},\left\{\pi(d) k q_{1} x_{1}\right\}\right)\right)
\end{aligned}
$$

with a suitable $\kappa_{1} \in F_{+}^{\times}$. Let $\left(V_{1}^{\prime \prime}, v_{1}^{\prime \prime}, \phi_{1}^{\prime \prime}\right)\left(\right.$ resp. $\left.\left(V_{1}^{*}, v_{1}^{*}, \phi_{1}^{*}\right)\right)$ be a moduli-system for $\Sigma\left(\hat{\Omega}^{\prime \prime}\right)\left(\right.$ resp. $\left.\Sigma\left(\hat{\Omega}^{*}\right)\right)$. Embed $V_{T_{1}}$ (resp. $\left.V_{U_{1}}\right)$ in $V_{1}^{\prime \prime}\left(\right.$ resp. $\left.V_{1}^{*}\right)$ in such a way that $\phi_{T_{1}}=\phi_{1}^{\prime \prime} \circ j_{1}$ (resp. $\left.\phi_{U_{1}}=\phi_{1}^{*} \circ j_{1}\right)$. We have a biregular isomorphism $\psi^{\prime \prime}$ (resp. $\psi^{*}$ ) of $V_{1}^{\prime \prime}\left(\right.$ resp. $\left.V_{1}^{*}\right)$ onto $V^{\prime \prime \tau}\left(\right.$ resp. $\left.V^{* \tau}\right)$ over $M_{1 c}$ which induces the isomorphism $\psi_{T}$ (resp. $\psi_{U}$ ) of $V_{T_{1}}$ (resp. $V_{U_{1}}$ ) to $V_{T}^{\tau}$ (resp. $V_{U}^{\tau}$ ).

We have $\hat{\Omega}^{*} \sim \Omega^{* \tau} \sim \Omega^{\prime \prime \sigma \tau} \sim \Omega^{\prime \prime \tau \sigma_{1}} \sim \Omega^{\prime \prime \sigma_{1}}$. Therefore, there is a biregular isomorphism $J_{1}$ of $V_{1}^{*}$ to $V_{1}^{\prime \prime \sigma_{1}}$ such that $v_{1}^{\prime \prime \sigma_{1}}=J_{1} \circ v_{1}^{*}$. We can identify $J_{V_{1} T_{1}}\left(u_{1}\right)$ with the restriction of $J_{1}$ to $V_{T_{1}}$.

Now we have

$$
J^{\tau} \circ \psi^{*} \circ v_{1}^{*}=J^{\tau} \circ v^{* \tau}=\left(J \circ v^{*}\right)^{\tau}=v^{\prime \prime \sigma \tau}
$$

on one hand, and

$$
\psi^{\prime \prime \sigma_{1}} \circ J_{1} \circ \varkappa_{1}^{*}=\psi^{\prime \prime \sigma_{1}} \circ \varkappa^{* \sigma_{1}}=\left(\psi^{\prime \prime} \circ v^{*}\right)^{\sigma_{1}}=\nu^{\prime \prime \tau \sigma_{1}}=v^{\prime \prime \sigma \tau}
$$


on the other. Therefore $J^{\tau} \circ \psi^{*}=\psi^{\prime \prime \sigma_{1}} \circ J_{1}$. It follows that

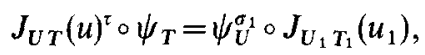

where $\sigma_{1}=\rho_{H_{1}}\left(u_{1}\right)$.

3.9. We prove that $\psi_{U}$ is defined over $k_{U_{1}} H_{1}$. Let $\pi_{1}$ be an automorphism of $M_{1 c}$ over $k_{U_{1}} H_{1}$. By [CI. 3.3,3.5], there is $u \in U \cap \mathscr{G}_{H+}$ such that $\rho_{H}(u)=\tau \pi_{1} \tau^{-1}$ on $M_{c}$. Put $u_{1}=u(u)$. Then $\rho_{H_{1}}\left(u_{1}\right)=\pi_{1}, J_{U U}(u)=\mathrm{id}$., and $J_{U_{1} U_{1}}\left(u_{1}\right)=\mathrm{id}$. Hence by (3.8.1) we have $\psi_{U}^{\pi_{1}}=\psi_{U}$. This being true for all $\pi_{1} \in \operatorname{Gal}\left(M_{1 c} / k_{U_{1}} H_{1}\right), \psi_{U}$ is defined over $k_{U_{1}} H_{1}$.

Therefore, for all $U \in \mathscr{W}$ and $U_{1}=u(U)$, we have a biregular isomorphism $\psi_{U}$ : $V_{U_{1}} \rightarrow V_{U}^{\tau}$ over $k_{U_{1}} H_{1}$ satisfying (3.8.1), i.e. we have proved the main Theorem for $\left\{V_{U}\right.$, $\left.\phi_{U}, J_{U T}(u) \mid U, T \in \mathscr{W}, u \in \mathscr{G}_{H+}\right\}$ over $H$. Going through the reduction process of [CI, II], we conclude that $u$ satisfies all the conditions of Theorem 1.3.

\section{Conjugations of Miyake's Models}

4.1. Let $K$ be a totally imaginary quadratic extension of a totally real algebraic number field $F$ of degree $g$, and $B$ a central simple algebra over $K$ with a positive involution $\rho$ which coincides with the complex conjugation on $K$. Choose $g$ embeddings $\tau_{1}, \ldots, \tau_{g}$ of $K$ into $C$ so that their restrictions to $F$ are all distinct. Put $n^{2}=[B: K]$. Decompose $B_{\mathbf{R}}=B \otimes_{\mathbf{Q}} \mathbf{R}$ into the direct sum of simple algebras $B_{1} \oplus \cdots$ $\oplus B_{g}$. Let $l_{\lambda}$ be the identity element of $B_{\lambda}$. For each $\lambda=1, \ldots, g$, there is an $R$-linear isomorphism $\phi_{\lambda}$ of $B_{\lambda}$ onto $M_{n}(C)$ such that $\phi_{\lambda}\left(x^{\rho}\right)={ }^{t} \overline{\phi_{\lambda}(x)}$ for all $x \in B_{\lambda}$. Fix $\phi_{\lambda}$ once and for all. For a $\rho$-hermitian element $h$ of $B^{\times}$, i.e. an element $h$ of $B^{\times}$such that $h^{\rho}$ $=h$, denote the signature of $\phi_{\lambda}(h)$ by $J_{\lambda}(h)$.

Let $\delta$ be an involution of $B$ which coincides with $\rho$ on $K$. Define an algebraic group $G$ over $\mathbf{Q}$ so that the $\mathbf{Q}$-rational points of $G$ are

$$
G_{\mathbf{Q}}=\left\{\alpha \in B^{\times} \mid \alpha \alpha^{\delta}=v(\alpha) \in F^{\times}\right\} .
$$

The semi-simple part of $G$ is

$$
G^{u}=\{\alpha \in G \mid v(\alpha)=1 \text { and } N(\alpha)=1\},
$$

where $N$ denotes the reduced norm of $B$ to $K$. Denote the homogeneous space $G_{\mathbf{R}}^{u}$ modulo a maximal compact subgroup by $\mathscr{H}$.

Fix a $\rho$-hermitian element $h \in B^{\times}$such that $x^{\delta}=h x^{\rho} h^{-1}$ for all $x \in B$. Let $J_{\lambda}$ $=J_{\lambda}(h)=(r(\lambda), s(\lambda))$. Put

$$
J_{r(\lambda), s(\lambda)}=\left[\begin{array}{cc}
1_{r(\lambda)} & 0 \\
0 & -1_{s(\lambda)}
\end{array}\right] .
$$

As in $[3,1.4]$, consider an element $j$ of $B_{\mathbf{R}}$ such that $j^{\delta}=-j, j^{2}=-1$ and such that $\left\{u \in G_{\mathbf{R}}^{u} \mid u j=j u\right\}$ is a maximal compact subgroup of $G_{\mathbf{R}}^{u}$. By [3, Corollary 1, Prop. 2], for each $\lambda=1, \ldots, g$, there is an isomorphism $\omega_{\lambda}$ of $B_{\lambda}$ onto $M_{n}(\mathbf{C})$ such that

$$
\omega_{\lambda}\left(x^{\delta}\right)=J_{r(\lambda), s(\lambda)} \overline{\omega_{\lambda}(x)} J_{r(\lambda), s(\lambda)} \text { for all } x \in B_{\lambda},
$$


and

$$
\omega_{\lambda}\left(j t_{\lambda}\right)=\sqrt{-1} J_{r(\lambda), s(\lambda)} .
$$

Using $\omega_{\lambda}$ 's, we can identify $\mathscr{H}$ with the product $\prod_{\lambda=1}^{g} \mathscr{H}_{r(\lambda), s(\lambda)}$, where $\mathscr{H}_{r, s}$ denotes the bounded symmetric domain consisting of all $r \times s$ complex matrices $z$ such that $1_{r}$ $-z^{t} \bar{z}$ is positive hermitian. In this way we have a bounded symmetric domain structure on $\mathscr{H}$. The main Theorem of [3] states that there is a canonical system of models for the quotients of $\mathscr{H}$ by arithmetic subgroups of $G$.

4.2. Let $\tau$ be an automorphism of C. For each $\lambda=1, \ldots, g$, there is a unique $\mu$ so that $\tau_{\mu} \tau=\tau_{\lambda}$ on $F$. Put $\sigma(\lambda)=\mu$. By a Theorem of Landherr [2] on hermitian forms over division algebras, there is a $\rho$-hermitian $h_{1}$ of $B^{\times}$such that i) for every finite prime $\not h$ of $F$, there is $x_{\not h} \in B_{p h}^{\times}$such that $h_{1}=x_{h h} h x_{p h}^{o}$; and ii) $J_{\lambda}\left(h_{1}\right)=J_{\sigma(\lambda)}(h)$. Let $\delta_{1}$ be the involution of $B$ given by $x \mapsto h_{1} \times h_{1}^{-1}$. Then $\left(B, \delta_{1}\right)$ defines a reductive group $G_{1}$ as in 4.1.

Since $J_{\lambda}\left(h_{1}\right)=J_{\sigma(\lambda)}(h)$, there is an R-linear isomorphism $\mathfrak{u}_{\lambda}$ of $B_{\lambda}$ to $B_{\sigma(\lambda)}$ such that $\mathfrak{u}_{\lambda}\left(x^{\delta_{1}}\right)=\mathfrak{u}_{\lambda}(x)^{\delta}$. Putting all $\mathfrak{u}_{\lambda}$ 's together, we get an $R$-linear automorphism $\mathfrak{u}_{x}$ of $B_{\mathbf{R}}$ so that $\mathfrak{u}_{\infty}\left(x^{\delta_{1}}\right)=\mathfrak{u}_{x}(x)^{\delta}$ for all $x \in B_{\mathbf{R}}$. The automorphism $\mathfrak{u}_{x}$ induces an isomorphism between $G_{\mathbf{R}}^{u}$ and $G_{1 \mathbf{R}}^{u}$.

Put $\varepsilon_{\lambda}=1$ if $\tau_{\lambda}=\tau_{\sigma(\lambda)} \tau$ on $K$, and $\varepsilon_{\lambda}=-1$ if $\tau_{\lambda}=\tau_{\sigma(\lambda)} \tau \rho$ on $K$. Let $\varepsilon=\varepsilon_{1} l_{1}+\cdots$ $+\varepsilon_{g} l_{g} \in B_{R}$. Denote by $j_{1}$ the unique element of $B_{\mathbf{R}}$ such that $\mathfrak{u}_{x}\left(j_{1} \varepsilon\right)=j$. Then we have $j_{1}^{\delta_{1}}=-j_{1}, j_{1}^{2}=-1$ and $\left\{u_{1} \in G_{1 \mathbf{R}}^{u} \mid u_{1} j_{1}=j_{1} u_{1}\right\}$ is a maximal compact subgroup of $G_{1 \mathbf{R}}^{u}$. Hence $j_{1}$ defines an isomorphism $\omega_{1 \lambda}$ of $B_{\lambda}$ to $M_{n}(\mathbf{C})$ for each $\lambda=1, \ldots, g$, as in 4.1. Using the $\omega_{1 \lambda}$ 's we can make the quotient of $G_{1 \mathbf{R}}^{u}$ modulo a maximal compact subgroup into a bounded symmetric domain $\mathscr{H}_{1}$. Let $\left\{V_{X_{1}}, \phi_{X_{1}}, J_{W_{1} X_{1}}\left(u_{1}\right)\right\}$ be a system of canonical models for the quotients of $\mathscr{H}_{1}$ by arithmetic subgroups of $G_{1}$. Then the models $\left\{V_{X}, \phi_{X}, J_{W X}(u)\right\}$ associated with $G$ and the models $\left\{V_{X_{1}}, \phi_{X_{1}}, J_{W_{1} X_{1}}\left(u_{1}\right)\right\}$ are related in the way described in Theorem 1.3. This fact can be proved in a similar way. Actually this is the easier case, because the bounded symmetric domains in question parametrize families of abelian-varieties themselves.

4.3. We make some comments on the special case where $\tau$ is the complex conjugation. In this case we have $G_{1}=G$. Note that the domains $\mathscr{H}_{1}$ and $\mathscr{H}$ are not equivalent unless $r(\lambda)=s(\lambda)$ or $r(\lambda) \cdot s(\lambda)=0$ for each $\lambda$.

The isomorphism $\mathfrak{u}$ in the main Theorem can be given by $u \mapsto \alpha^{-1} u \alpha$ with a "negative" element $\alpha$ of $G_{Q}$ in the sense of $[4, \S 3]$. This fact follows easily from the results of $[4, \S 3]$. The finite part $\alpha_{0}$ of $\alpha$ belongs to the group $\mathscr{G}_{j+}$ defined in $[3,3.4]$ if and only if $r(\lambda)=s(\lambda)$ for all $\lambda$. When this happens we have $\bar{\phi}_{T}(\alpha(\bar{z}))$ $=J_{T T_{1}}\left(\alpha_{0}\right) \circ \phi_{T_{1}}(z)$ for all $T \in 3$ and $T_{1}=\mathfrak{u}(T) \in \mathcal{Z}_{1}$. The corresponding fact for Shimura's models is much harder to prove, see [4].

\section{References}

1. Doi, K., Naganuma, H.: On the algebraic curves uniformized by arithmetical automorphic functions. Ann. Math. 86, $449-460$ (1967)

2. Landherr, W.: Äquivalenz Hermitescher Formen über beliebige algebraische Zahlkörper. Abh. Hamb. pp. 245-248 (1935) 
3. Miyake, K.: Models of certain automorphic function fields. Acta Math. 126, 245-307 (1971)

4. Shih, K.: Anti-holomorphic automorphisms of arithmetic automorphic function fields. Ann, of Math. 103, 81-102 (1976)

5. Shimura, G.: On analytic families of polarized abelian varieties and automorphic functions. Ann. Math. 78, 149-192 (1963)

6. Shimura, G.: On the field of definition for a field of automorphic functions: I, II, III. Ann. Math. 80, 160-189 (1964); 81, 124-165 (1965); 83, 377-385 (1966)

7. Shimura, G.: Moduli and fibre systems of abelian varieties. Ann. Math. 83, 294-338 (1966)

8. Shimura, G.: Construction of class fields and zeta functions of algebraic curves. Ann. Math. 85, 58$159(1967)$

9. Shimura, G.: Discontinuous groups and abelian varieties. Math. Ann. 168, 171-199 (1967)

10. $=$ [A] Shimura, G.: Algebraic number fields and symplectic discontinuous groups. Ann. of Math. 86, 503-592 (1967)

11. $=[\mathrm{C}]$ Shimura, G.: On canonical models of arithmetic quotients of bounded symmetric domains: I, II. Ann. Math. 91, 144-222 (1970); 92, 528-549 (1970)

Received January 6, 1977 